\title{
Avaliação da eficiência dos serviços de saneamento básico no combate às endemias nos municípios do Estado do Tocantins ${ }^{1}$
}

Assessment of the efficiency of the basic sanitation services on the combat to the endemics in the counties of the State of Tocantins

\author{
Adriano Araújo Firmino Valdevino 2 \\ Júlio César Lima Medeiros ${ }^{3}$ \\ Adriano Paixão Nascimento ${ }^{4}$ \\ Aurélio Picanço Pessôa ${ }^{5}$
}

\begin{abstract}
Resumo: O combate a endemias relacionadas à condição de vida (tais como a diarréia, cólera, leptospirose, tracoma etc.) constitui-se uma das prioridades da atual política de saúde do SUS. Muitas destas endemias estão relacionadas à precariedade ou falta de serviços de saneamento básico. Frente às evidências do beneficio dos serviços de saneamento sobre a Saúde Pública, torna-se necessário que o planejamento e a execução de políticas de saúde e de intervenções a partir do saneamento sejam considerados de forma mutua e compassada, constituindo-se uma estratégia de Saúde Pública preventiva e social. O objetivo deste trabalho é avaliar os serviços de saneamento básico dos municípios tocantinenses quanto a sua eficiência no combate a endemias relacionadas à falta ou precariedade desses serviços, especialmente às endemias de veiculação hídrica. Parte-se da hipótese de que o número de casos dessas doenças está negativamente relacionado com as condições verificadas na área de saneamento básico. Para realização desse estudo foi utilizada a Metodologia da Análise Envoltória de Dados (DEA) para avaliar a eficiência dos municípios no combate às endemias a partir dos serviços de saneamento básico em 2006. Os resultados obtidos apontam para uma realidade caracterizada pela precariedade dos serviços de saneamento básico.
\end{abstract}

Palavras-Chave: Saneamento Básico; Saúde Pública; Análise Envoltória de Dados.

\begin{abstract}
The endemic combat related to the life conduction (each as, diarrhea, leptospirosis, cholera, trachoma etc.) is the priority of the actual health politic of SUS. Many of this endemics are related to the precariousness or no services of basic sanitation. In front of the benefits evidences of the sanitation services in Public Health, it become necessary that the planning and the acting of the health politics and the intervention starting from the sanitation are considered in both ways and compassed, being an strategy of Public Health preventive and social. The objective of this work is evaluate the basic sanitation services of the Tocantins' counties as its efficiency to the endemics combat related to the lack or precariousness of this services, specially the endemics of hydrics linked. Starts from the hypothesis of the number of cases of this diseases is negativity related with the conditions verified in the basic sanitation area. To the realization of this study it has been used the Methodology Data Envelopment Analysis (DEA) to evaluate the efficiency of the counties on the combat to the endemics starting from the services of basic sanitation in 2006. The results have pointed to a reality characterized by the precariousness of the services in basic sanitation.
\end{abstract}

Key Words: Sanitation Services; Public Health; Data Envelopment Analysis.

JEL: Q25; C81.

\footnotetext{
${ }^{1}$ Artigo recebido em abril de 2010 e aprovado em setembro de 2010.

${ }^{2}$ Economista pela UFPB. Mestre e Doutor em Economia (PIMES/UFPE). Professor adjunto do Curso de Ciências Econômicas e do Programa de Pós-Graduação em Desenvolvimento Regional da UFT. Email: afva77@uft.edu.br

3 Economista pela UFT. Mestrando do Programa de Pós-Graduação em Desenvolvimento Regional da UFT. E-mail: juliomedeiros@uft.edu.br

4 Doutor em Economia Aplicada pela UFV. Professor Adjunto do Curso de Ciências Econômicas da UFT. E-mail: anpaixao@uft.br

5 Doutor em Hidráulica e Saneamento pela Escola de Engenharia de São Carlos. Professor Adjunto do

Curso de Engenharia Ambiental da UFT. E-mail: aureliopicanco@uft.edu.br
} 


\section{Introdução}

Desde o final do século passado o Brasil vem enfrentando alguns problemas relacionados ao processo acelerado de urbanização verificado em diversas regiões. Aumento da criminalidade, crescimento populacional desordenado, falta de infraestrutura básica e dificuldades em fornecer serviços públicos essenciais, entre outros, são problemas cada vez mais presentes no dia-a-dia. No que diz respeito à infraestrutura, parece ser consenso que os principais desafios dos gestores de políticas públicas dizem respeito ao fornecimento de serviços de saneamento.

A intervenção governamental no setor de saneamento básico é justificada por pelo menos dois motivos. Em primeiro lugar, este setor é caracterizado pela existência de custos fixos altos, típico de um monopólio natural 6 . Em segundo lugar, os serviços de saneamento básico envolvem externalidades positivas, haja vista que pode reduzir o número de casos de doenças de veiculação hídrica e doenças relacionadas à condição de vida7.

A relação entre os serviços de saneamento básico e Saúde Pública é evidenciada na própria definição de saneamento. Segundo a Organização Mundial de Saúde - OMS (apud HELLER, 1998), "saneamento constitui o controle de todos os fatores do meio físico do homem, que exercem ou podem exercer efeitos deletérios sobre seu estado de bem estar físico, mental ou social” (HELLER, 1998, p. 74-75). No campo da pesquisa empírica, dados da Fundação Nacional de Saúde - FUNASA (2006) apontam evidências de que no Brasil as doenças resultantes da falta ou inadequação de saneamento, especificamente em áreas pobres, têm agravado o quadro epidemiológico. Ainda segundo a FUNASA (2006), para cada $\mathrm{R} \$ 1,00$ (um real) investido no setor saneamento, economiza-se cerca de $\mathrm{R} \$ 4,00$ (quatro reais) na área de medicina curativa. As conseqüências desta relação são apontadas por Carmo \& Távora Júnior (2004). Segundo estes autores, os problemas gerados pela precariedade de serviços de saneamento básico são visíveis nos altos custos em tratamentos hospitalares de endemias relacionadas principalmente a água e esgoto não tratados.

Contudo, Heller (1998) chama a atenção para o fato de que as evidências empíricas da relação benéfica entre saneamento e Saúde Pública carecem de estudos mais específicos. Neste tocante, o autor levanta algumas lacunas, tais como:

- Distinção das populações sob risco a partir de cenários distintos de ausência de condições adequadas de saneamento;

- Efeitos das intervenções por meio do saneamento sobre um conjunto mais amplo de indicadores;

- Efeitos de intervenções isoladas e conjugadas por meio de saneamento; e

\footnotetext{
${ }^{6}$ Sobre aspectos teóricos relacionados à regulamentação governamental em monopólios naturais ver Pindyck \& Rubinfeld (2002); Varian (1992 e 2003), Vasconcellos \& Oliveira (2006) e Kupfer \& Hasenclever (2002).

7 Segundo a Organização Mundial de Saúde - OMS (apud Universidade IMES, 2008), as doenças relacionadas com a água, por exemplo, podem ser classificadas em três grupos, segundo a forma de contaminação: doenças causadas pela ingestão de água contaminada (cólera, disenteria, febre tifóide, giardise, hepatite infecciosa, leptospirose etc.), doenças causadas pelo contato com água contaminada (tracoma, verminose, esquistossomose etc.) e doenças transmitidas por insetos que se desenvolve na água (dengue, febre amarela, malária etc.).
} 

saneamento ${ }^{8}$.

- Quantificação dos riscos relacionados à falta ou precariedade de serviços de

Soares, Bernardes \& Cordeiro Netto (2002) apontam para o fato de que algumas intervenções a partir do saneamento podem provocar efeitos deletérios. Por exemplo, alguns subprodutos do tratamento de água a partir do uso do cloro podem provocar o aumento na incidência de certos tipos de câncer. Mesmo tratamentos alternativos ao cloro resultam em subprodutos que pode afetar de forma negativa a saúde humana. Adicionalmente, intervenções a partir de saneamento podem provocar impactos ambientais consideráveis que, em última instância, podem afetar de forma negativa a saúde humana (SOARES, BERNARDES \& CORDEIRO NETTO, 2002).

As considerações apresentadas anteriormente, longe de invalidar a idéia de que o saneamento promove benefícios para a Saúde Pública, apresentam aspectos relevantes que devem ser levados em consideração em estudos na área, sugerindo possíveis programas de pesquisas. O benefício causado pelo saneamento na área de saúde é amplamente reconhecido por pesquisadores da área (SOARES, BERNARDES \& CORDEIRO NETTO, 2002).

Outro aspecto relevante dos serviços de saneamento diz respeito à relação entre saneamento e desenvolvimento econômico. Segundo Heller (1998), países com elevados graus de desenvolvimento, em geral, apresentam menores carências em relação ao fornecimento de serviços de saneamento diante das necessidades sociais. Segue-se, ainda segundo este autor, que os países com maiores coberturas nos serviços de saneamento têm populações mais saudáveis.

No Brasil, o reconhecimento do fato de que as políticas na área de Saúde Pública devem considerar uma ótica preventiva e ações de caráter social resultou na criação do Sistema Único de Saúde (SUS) a partir da Constituição de 1988. Segundo Buss (2000), estratégias como as inovações implementadas nos Programa de Agentes Comunitários e Saúde da Família e a ampliação do piso assistencial básico consistem em avanços na promoção de saúde, gerando ganhos consideráveis, não só relacionados à saúde, mas também à condição de vida da população. Adicionalmente, estratégias de desenvolvimento local, tal como a Agenda 21, podem favorecer ações pró-saúde (BUSS, 2000). Outro avanço, apontado por Santos \& Westphal (1999), foi a descentralização das políticas de Saúde Pública, privilegiando o município na implantação de políticas de promoção à saúde.

Embora haja o reconhecimento de que a legislação brasileira incorporou os novos paradigmas relacionados à Saúde Pública, observa-se na prática um descompasso entre as políticas de saneamento e as estratégias públicas em Saúde Pública. Segundo Heller (1998), dois fatores indicavam este descompasso. Por um lado, as práticas e planejamentos na área de saneamento não consideravam os benefícios na Saúde Pública. Por outro lado, as políticas de Saúde Pública tinham caráter exclusivamente curativo, excluindo um papel mais ativo das intervenções via saneamento (HELLER, 1998; SANTOS \& WESTPHAL, 1999; SANTOS, 2007).

Uma das prioridades da atual política de saúde do SUS é o combate de endemias relacionadas à condição de vida, tais como a dengue, diarréia, malária, hanseníase, cólera, leptospirose, tracoma etc. Muitas destas endemias estão relacionadas à precariedade ou falta de serviços de saneamento básico. Diante da exposição apresentada aqui, fica evidente e as avaliações de tais políticas devem

\footnotetext{
${ }^{8}$ Segundo Heller (1998), as tentativas de quantificação de riscos apresentam grande dispersão. Isso seria resultado da diversidade de realidades estudadas.
} 
considerar aspectos relacionados com a provisão e qualidade dos serviços de saneamento básico.

Diante disto, o objetivo deste trabalho é avaliar os serviços de saneamento básico dos municípios tocantinenses quanto a sua eficiência no combate de endemias relacionadas à falta ou precariedade desses serviços, especialmente às endemias de veiculação hídrica. Parte-se, portanto, da hipótese de que investimentos e gastos em saneamento provocam a redução nos casos dessas doenças. No entanto, esta relação pode não ser a mesma para todos os municípios analisados, cabendo uma análise de eficiência. Para realização desse estudo foi utilizada a Metodologia da Análise Envoltória de Dados (DEA) para analisar a eficiência em 2006.

Por fim, ressalta-se que não é objetivo desta pesquisa verificar a validade da hipótese de que há uma relação causal (negativa) entre condições verificadas de saneamento e número de casos de certas endemias.

\section{Metodologia}

A análise da eficiência relativa de unidades produtivas pode ser realizada a partir de duas abordagens: paramétrica e não-paramétrica. As abordagens paramétricas utilizam instrumentais econométricos para estimar uma função de produção estocástica. Estas abordagens necessitam da especificação das formas funcionais das funções de produção estimadas e, geralmente, utilizam medidas de tendência central para analisar a eficiência relativa das unidades produtivas.

As abordagens não paramétricas utilizam de programação matemática para construir fronteiras de eficiências determinísticas. Em geral, estes métodos utilizam programação linear para construir fronteiras lineares por partes. A eficiência das unidades produtivas é determinada a partir de medidas radiais e de funções de distância. O Método da Análise Envoltória dos Dados (DEA) se enquadra nesta abordagem. Adota-se como pressuposto que a forma de produção adotada pelas unidades produtivas mais eficientes pode ser copiada pelas unidades produtivas menos eficientes (CHARNES et al., 1994).

\subsection{Análise envoltória de dados - DEA}

A idéia central do método DEA é encontrar a melhor unidade "virtual"9 para cada unidade real. Se a unidade "virtual" conseguir produzir maiores quantidades de produtos, utilizando-se a mesma ou menor quantidade de insumos, então a unidade de produção real será ineficiente (LINS \& MEZA, 2000; GOMES \& BAPTISTA 2004). Segundo Lins \& Calôba (2006), o método DEA possui algumas características que podem representar vantagens em relação a outros métodos de análise de eficiência:

i) Não é necessário transformar os insumos em unidades monetárias;

ii) Os índices de eficiência são construídos a partir de dados reais;

iii) Considera a possibilidade de que os benchmarks ${ }^{10}$ representem outiliers para as demais unidades produtivas;

\footnotetext{
9 A unidade virtual refere-se à combinação que projeta a unidade produtiva na fronteira de eficiência. $10 \mathrm{O}$ termo benchmark refere-se às unidades produtivas eficientes que podem ser tomadas como referência para as unidades produtivas ineficientes.
} 
iv) Além de identificar as unidades produtivas eficientes, mede e localiza as unidades produtivas ineficientes a partir de uma função de produção linear por partes;

v) Determina a eficiência relativa das unidades produtivas, contemplando cada uma destas unidades relativamente a todas as outras que compõem o grupo estudado;

vi) Fornece os benchmarks para as unidades produtivas ineficientes;

vii) A fronteira de eficiência obtida representa um conjunto de unidades produtivas Pareto-Eficientes; e

viii) Não necessidade da determinação de uma forma funcional para a fronteira de eficiência.

A escolha pela utilização da metodologia DEA para a análise de eficiência dos municípios tocantinenses no combate às endemias de veiculação hídrica a partir dos serviços de saneamento é justificada pelas vantagens apresentadas. Adicionalmente, optou-se por adotar uma análise com orientação produto. Desta forma, é possível calcular o número de casos de doenças de veiculação hídrica que um dado município ineficiente deveria apresentar, dado a quantidade de insumos utilizados, para ser considerado eficiente.

A construção de uma fronteira de eficiência a partir da metodologia DEA pode ser feita considerando retornos constantes de escala ou retornos variados de escala (crescentes e decrescentes). O modelo com retornos constantes de escala é conhecido como DEA-CCR, e considera o axioma da proporcionalidade entre produtos e insumos. O modelo com retornos variados de escala é conhecido como DEA-BCC e considera o axioma da convexidade. Graficamente,

\section{Gráfico 1 - Fronteiras de Eficiências nos Modelos DEA-CCR e DEA-BCC}

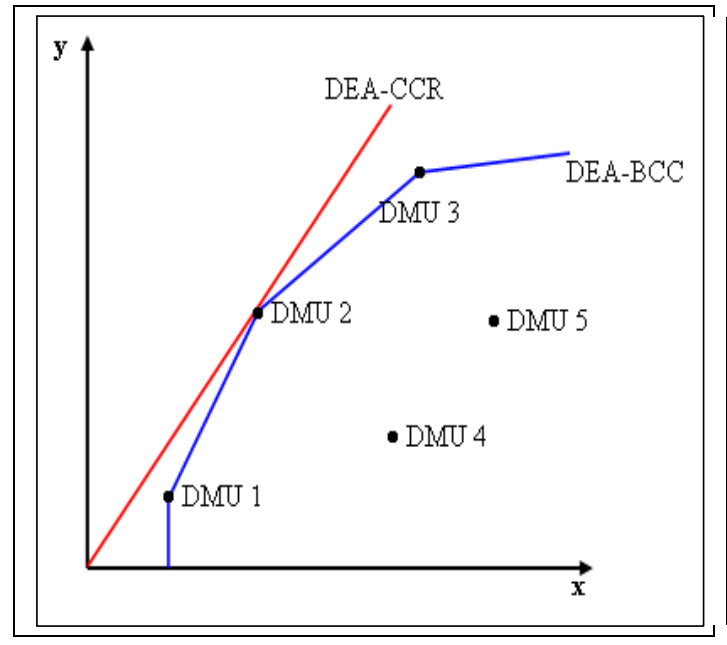

Fonte: Elaboração própria.

Considere a existência de $\mathrm{k}$ insumos e $\mathrm{m}$ produtos para cada uma das $\mathrm{n}$ $\mathrm{DMUs}^{11}$. As matrizes a seguir representam essas unidades:

${ }^{11}$ Do inglês Decision Marking Units. Este termo refere-se às unidades produtivas, sendo amplamente utilizado nas pesquisas com DEA. 


$$
\mathrm{X}=\left(\begin{array}{cccc}
\mathrm{x}_{11} & \mathrm{x}_{12} & \cdots & \mathrm{x}_{1 \mathrm{n}} \\
\mathrm{x}_{21} & \mathrm{x}_{22} & \cdots & \mathrm{x}_{2 \mathrm{n}} \\
\vdots & \vdots & \ddots & \vdots \\
\mathrm{x}_{\mathrm{k} 1} & \mathrm{x}_{\mathrm{k} 2} & \cdots & \mathrm{x}_{\mathrm{kn}}
\end{array}\right)_{\mathrm{k} \times \mathrm{n}} \quad \mathrm{Y}=\left(\begin{array}{cccc}
\mathrm{y}_{11} & \mathrm{y}_{12} & \cdots & \mathrm{y}_{1 \mathrm{n}} \\
\mathrm{y}_{21} & \mathrm{y}_{22} & \cdots & \mathrm{y}_{2 \mathrm{n}} \\
\vdots & \vdots & \ddots & \vdots \\
\mathrm{y}_{\mathrm{ml}} & \mathrm{y}_{\mathrm{m} 2} & \cdots & \mathrm{y}_{\mathrm{mn}}
\end{array}\right)_{\mathrm{m} \times \mathrm{n}}
$$

$\mathrm{Na}$ matriz X cada linha representa um insumo e cada coluna representa uma DMU. Na matriz Y cada linha representa um produto e cada coluna representa uma DMU. A medida de eficiência para cada DMU é dada por:

$$
\text { Eficiência da DMU d }=\mathrm{Ef}_{\mathrm{d}}=\frac{\mu^{\prime} \mathrm{y}_{\mathrm{id}}}{v^{\prime} \mathrm{x}_{\mathrm{jd}}}=\frac{\mu_{1} \mathrm{y}_{1 \mathrm{~d}}+\mu_{2} \mathrm{y}_{2 \mathrm{~d}}+\ldots+\mu_{\mathrm{m}} \mathrm{y}_{\mathrm{md}}}{v_{1} \mathrm{y}_{1 \mathrm{~d}}+v_{2} \mathrm{y}_{2 \mathrm{~d}}+\ldots+v_{\mathrm{k}} \mathrm{y}_{\mathrm{kd}}}
$$

Onde $\square$ é um vetor (m x 1) de pesos associados aos produtos e $\square$ é um vetor ( $\mathrm{k}$ $\mathrm{x}$ 1) de pesos associados aos insumos.

Segundo LINS \& MEZA (2000), o modelo DEA-CCR com orientação produto corresponde ao seguinte problema de programação linear:

$$
\begin{aligned}
& \min _{\mu, v} \phi_{\mathrm{d}}=\frac{v^{\prime} \mathrm{x}_{\mathrm{id}}}{\mu^{\prime} \mathrm{y}_{\mathrm{jd}}} \\
& \text { s.a.: } \\
& \frac{v^{\prime} \mathrm{x}_{\mathrm{ik}}}{\mu^{\prime} \mathrm{y}_{\mathrm{jk}}} \geq 1 \forall \mathrm{k} \\
& \mu_{\mathrm{i}}, v_{\mathrm{j}} \geq 0 \forall \mathrm{i}, \mathrm{j} \\
& \text { Onde } \phi_{\mathrm{d}}=\frac{1}{\mathrm{Ef}_{\mathrm{d}}} .
\end{aligned}
$$

A equação (2.2) representa um Problema de Programação Fracionada (PPF), que deve ser resolvido para cada uma das DMUs analisadas [GOMES \& BAPTISTA (2004)]. Para uma representação por meio de um Problema de Programação Linear, é necessário que o denominador da função objetivo seja uma constante ${ }^{12}$. Geralmente, considera-se que o denominador seja igual à unidade. Portanto,

$$
\begin{aligned}
& \min _{\mu, v} \phi_{\mathrm{d}}=v^{\prime} \mathrm{x}_{\mathrm{id}} \\
& \text { s.a. : } \\
& \mu^{\prime} \mathrm{y}_{\mathrm{jd}}=1 \\
& \frac{v^{\prime} \mathrm{x}_{\mathrm{ik}}}{\mu^{\prime} \mathrm{y}_{\mathrm{jk}}} \geq 1 \quad \forall \mathrm{k} \\
& \mu_{\mathrm{i}}, v_{\mathrm{j}} \geq 0 \forall \mathrm{i}, \mathrm{j}
\end{aligned}
$$

A segunda restrição implica que

$$
\begin{aligned}
& v^{\prime} \mathrm{x}_{\mathrm{ik}} \geq \mu^{\prime} \mathrm{y}_{\mathrm{jk}} \forall \mathrm{k} \\
& v^{\prime} \mathrm{x}_{\mathrm{ik}}-\mu^{\prime} \mathrm{y}_{\mathrm{jk}} \geq 0 \forall \mathrm{k}
\end{aligned}
$$

A equação (2.3) é denominada de Modelos dos Multiplicadores, correspondendo à formulação primal do PPL para um DEA-CCR. A formulação dual do PPL, conhecido como Modelo do Envelope é expressa como:

\footnotetext{
${ }^{12}$ As restrições apresentadas no PPF expresso pela equação (2.2) não garante uma solução única. Por isso a necessidade de transformá-lo em um PPL (GOMES \& BAPTISTA, 2004).
} 


$$
\begin{aligned}
& \max _{\phi_{\mathrm{d}}, \lambda} \phi_{\mathrm{d}}=\frac{v^{\prime} \mathrm{x}_{\mathrm{id}}}{\mu^{\prime} \mathrm{y}_{\mathrm{jd}}} \\
& \text { s.a.: } \\
& -\phi_{\mathrm{d}} \mathrm{y}_{\mathrm{d}}+\lambda^{\prime} \mathrm{Y} \geq 0 \quad \forall \mathrm{i} \\
& \mathrm{x}_{\mathrm{d}}-\lambda^{\prime} \mathrm{X} \geq 0 \forall \mathrm{j} \\
& \lambda_{\mathrm{k}} \geq 0 \forall \mathrm{k}
\end{aligned}
$$

Onde $\lambda$ é o vetor ( $\mathrm{n} \times 1$ ) de pesos que correspondem à contribuição de cada DMU k $(\mathrm{k}=1, \ldots, \mathrm{n})$ formação do alvo da DMU d. Desta forma, as DMUs eficientes apresentaram um vetor nulo de $\lambda$. Por outro lado, as DMUs ineficientes apresentaram contribuições nulas para a formação do alvo de qualquer outra DMU ineficiente.

Considerando retornos variados de escala, o DEA-BCC na versão dual (Modelo do Envelope) com orientação produto é formulado a partir do seguinte PPL:

$$
\begin{aligned}
& \max _{\phi_{\mathrm{d}}, \lambda} \phi_{\mathrm{d}}=\frac{\nu^{\prime} \mathrm{x}_{\mathrm{id}}}{\mu^{\prime} \mathrm{y}_{\mathrm{jd}}} \\
& \text { s.a. : } \\
& -\phi_{\mathrm{d}} \mathrm{y}_{\mathrm{d}}+\lambda^{\prime} \mathrm{Y} \geq 0 \quad \forall \mathrm{i} \\
& \mathrm{x}_{\mathrm{d}}-\lambda^{\prime} \mathrm{X} \geq 0 \forall \mathrm{j} \\
& \mathrm{e}^{\prime} \lambda=1 \\
& \lambda_{\mathrm{k}} \geq 0 \forall \mathrm{k}
\end{aligned}
$$

Onde e é o vetor unitário ( $\mathrm{n} \times \mathrm{1}$ ). A formulação primal do DEA-BCC com orientação produto é:

$$
\begin{aligned}
& \min _{\mu, v} \phi_{\mathrm{d}}=v^{\prime} \mathrm{x}_{\mathrm{id}}+v^{*} \\
& \text { s.a.: } \\
& \mu^{\prime} \mathrm{y}_{\mathrm{jd}}=1 \\
& -v^{\prime} \mathrm{x}_{\mathrm{ik}}+\mu^{\prime} \mathrm{y}_{\mathrm{jk}}-v^{*} \leq 1 \quad \forall \mathrm{k} \\
& \mu_{\mathrm{i}}, v_{\mathrm{j}} \geq 0 \forall \mathrm{i}, \mathrm{j}
\end{aligned}
$$

Onde $\square^{*}$ é um fator de escala associado à restrição $e^{\prime} \lambda=1$. Se a tecnologia exibir retornos não-crescentes de escala, então $\mathrm{e}^{\prime} \lambda \leq 1$. No caso de retornos nãodecrescentes, tem-se e $e^{\prime} \lambda \geq 1$.

Considerando o DEA com orientação produto, o alvo de uma DMU consiste no nível de produção que deve ser alcançado, mantendo-se inalterados os níveis de utilização dos insumos, para que a DMU seja considerada eficiente. Esse alvo consiste na multiplicação entre o nível real de produção e o valor de $\phi$. Ou seja, a DMU deve aumentar sua produção em $(\phi-1) \cdot 100 \%$, mantendo seu nível de utilização de insumos, para atingir a fronteira de eficiência. Observa-se, portanto, que uma DMU eficiente apresentará $\phi=1$ e uma DMU ineficiente apresentará $\phi \geq 1$.

Por fim, cabe destacar o problema da baixa discriminação entre as DMUs analisadas, causada quando algumas das variáveis utilizadas beneficiam alguma das DMUs. O resultado é que algumas DMUs podem apresentar o que se chama de falsa eficiência, apresentada no Gráfico 2. Pode-se observar que a DMU 1, apesar de pertencer à fronteira de eficiência, não apresenta uma produção eficiente, haja vista que essa DMU pode aumentar sua produção mantendo constante o nível de utilização de insumos, caminhando em direção à DMU 2. Neste caso, diz-se que a DMU apresenta uma folga em sua produção, equivalente a sua distância em relação à DMU 
${ }^{13}$. A baixa discriminação resulta de empates que ocorrem entre DMUs com $100 \%$ de eficiência [MELLO et al., (2005)].

Gráfico 2 - Baixa Discriminação das DMUs no Modelo DEA-BCC: Falsa Eficiência

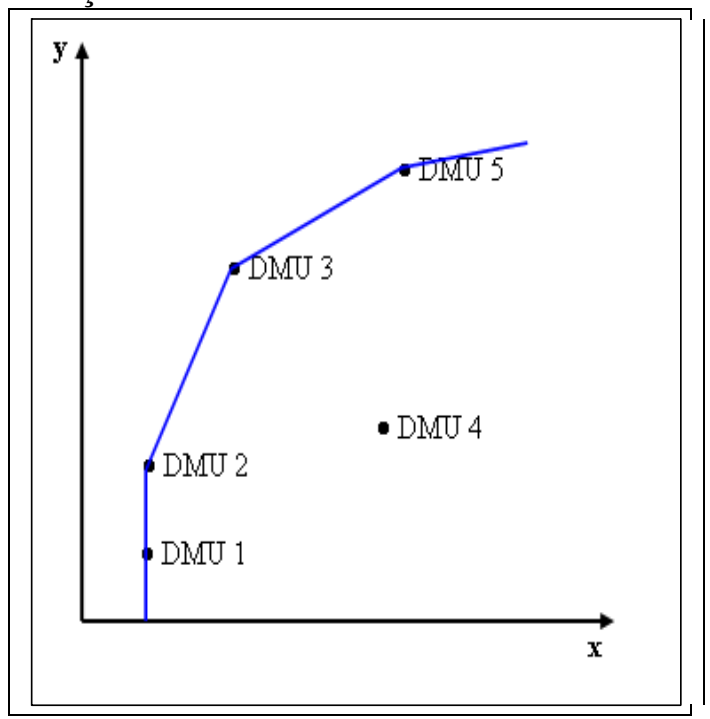

Fonte: Elaboração própria.

A baixa discriminação pode ser evitada a partir do uso do conceito de fronteira de eficiência inversa. Segundo Mello et al., (2005) e Leta et al., (2005), a fronteira de eficiência inversa consiste em uma avaliação pessimista da eficiência das DMUs, sendo composta pelas DMUs mais ineficientes. Esta fronteira é obtida fazendo a troca entre produtos e insumos. Graficamente,

Gráfico 3 - Fronteira Clássica e Fronteira Invertida no Modelo DEA-BCC

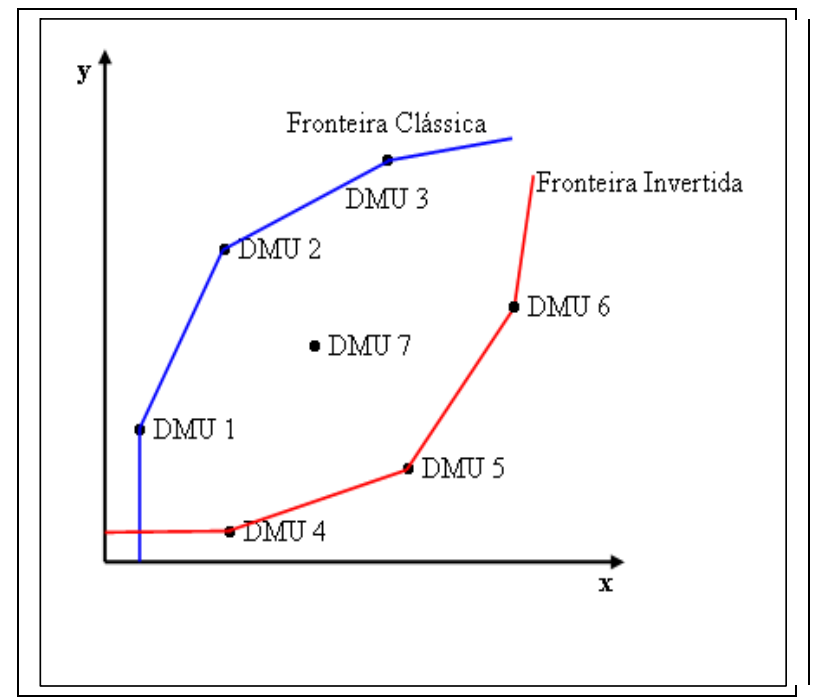

Fonte: Adaptado de MELLO et al., (2005).

${ }^{13}$ Em modelos DEA com a orientação insumo, o conceito de folga é similar, estando associado à possibilidade de uma DMU eficiente diminuir a utilização de insumos e manter o nível de produção. 
Segundo Leta et al., (2005), o problema da baixa discriminação pode ser evitado adotando-se o conceito de eficiência composta, definido como:

$$
E f_{\text {composta }}=\frac{E f_{\text {otimista }} \quad E f_{\text {pessimista }}+1}{2}
$$

Onde a eficiência otimista é obtida a partir da fronteira de eficiência clássica. Pode-se, ainda, definir um índice de eficiência normalizado, definido como:

$$
\mathrm{Ef}_{\text {normalizad a }}=\frac{\mathrm{Ef}_{\text {composta }}}{\mathrm{Ef}_{\text {máximo }}}
$$

Onde $\mathrm{Ef}_{\text {máximo }}$ refere-se ao valor calculado do maior índice de eficiência composta.

\subsection{Procedimentos adicionais}

Os resultados obtidos a partir da utilização do método DEA podem sofrer influências de DMUs consideradas outliers'4, especialmente o DEA-CCR. Para verificar a existência de outliers entre as DMUs analisadas nesta pesquisa, adotou-se a análise de clusters. Segundo MINGOTI (2005), dado um conjunto de variáveis ou características adotadas, a análise de clusters permite dividir uma amostra ou população em grupos, de tal forma que os elementos pertencentes ao mesmo grupo sejam homogêneos entre si e heterogêneos quando comparados com os elementos de outro grupo. A análise de clusters deve seguir as seguintes etapas:

i) Escolha de um critério de agrupamento das observações;

ii) Formação dos grupos a partir do critério escolhido;

iii) Definição do número de grupos, que pode ser realizado a posteriori, como resultado da análise, ou a priori, dado o conhecimento prévio sobre o problema;

iv) Validação do agrupamento, consistindo em uma etapa arbitrada ao pesquisador; e

v) Interpretação dos resultados.

Dado que o método DEA parte de distâncias radiais para o cálculo dos índices de eficiência, adotou-se o Método de Ligação Simples (single linkage), também conhecido como método do vizinho mais próximo. Considere dois grupos, $G_{i}$ e $G_{j}$, obtidos em uma determinada etapa do algoritmo. No Método de Ligação Simples, a distância entre os dois grupos é definida como:

$$
\mathrm{d}\left(\mathrm{G}_{\mathrm{i}}, \mathrm{G}_{\mathrm{j}}\right)=\min \left\{\left(\mathrm{x}_{\mathrm{i}}, \mathrm{x}_{\mathrm{j}}\right): \mathrm{i} \neq \mathrm{j}, \mathrm{x}_{\mathrm{i}} \in \mathrm{G}_{\mathrm{i}} \text { ex } \mathrm{x}_{\mathrm{j}} \in \mathrm{G}_{\mathrm{j}}\right\} \text {. }
$$

Ou seja, a similaridade entre dois grupos é definida a partir da distância entre os elementos extremos de cada grupo MINGOTI (2005).

O gráfico que demonstra a distância entre os elementos ou grupos em uma análise de clusters é chamado de dendrograma.

As análises de clusters realizadas nesta pesquisa partem da construção de dendrograma para os municípios tocantinenses, de modo a agregar tais municípios de acordo com suas características.

\footnotetext{
${ }_{14}$ Os outliers consistem em observações que apresentam consideráveis discrepâncias em relação à média.
} 


\section{Dados Utilizados}

Diante do objetivo central deste trabalho, avaliar a eficiência dos municípios no combate a determinadas endemias a partir dos serviços de saneamento básico, cada município tocantinense foi considerado como uma DMU.

Para compor a variável de produto (output), foram considerados os números de casos das doenças de veiculação hídrica apresentadas a seguir para o ano de 2006 por local de residência.

i) Doenças causadas pela ingestão de água contaminada: febre tifóide e leptospirose;

ii) Doenças causadas pelo contato com água contaminada: tracoma e esquistossomose; $\mathrm{e}$

iii) Doenças transmitidas por insetos que se desenvolve na água: dengue e malária.

As demais doenças de veiculação hídrica não foram consideradas por apresentarem incidência nula nos municípios tocantinenses no ano de 2006. Destaca-se que cerca de $90 \%$ do total de casos observados nos municípios analisados referem-se à dengue. $\mathrm{O}$ indicador utilizado foi o número total de casos das doenças apresentadas per capita. Adotou-se como variável de produto o inverso deste indicador, haja vista que a construção da fronteira de eficiência requer uma relação positiva entre insumos e produtos.

Os dados de endemias foram coletados no MINISTÉRIO DA SAÚDE (2008), através do Sistema de Informações de Agravos de Notificação. Os dados a respeito da população dos municípios foram obtidos no MINISTÉRIO DAS CIDADES (2008), a partir do Sistema Nacional de Informações sobre Saneamento (SNIS).

Em relação aos insumos (inputs), foram utilizadas variáveis relacionadas com os serviços de saneamento básico. Devido ao fato de que os municípios tocantinenses apresentam baixa ou nenhuma cobertura de esgotamento sanitário, drenagem, gestão de resíduos sólidos, as variáveis consideradas neste estudo estão associadas ao serviço de fornecimento de água tratada. As variáveis consideradas foram:

i) Ligações totais (ativas e inativas) para o ano de 2006;

ii) Volume de água produzido em 2006, expresso por 1.000m3/ano;

iii) Despesa total com produtos químicos em 2006, expressa em $\mathrm{R} \$$ /ano; $\mathrm{e}$

iv) Investimento realizado em 2005 com recursos próprios da prestadora de serviços, expresso em $\mathrm{R} \$$ /ano.

Segundo Soares et al., (2002) e Heller (1998), a saúde dos indivíduos é afetada pela quantidade e qualidade da água disponível para o consumo. As ligações totais e o volume de água produzido captam a disponibilidade de água para a população. A despesa total com produtos químicos capta os esforços para manter ou melhorar a qualidade da água fornecida. Por fim, o investimento realizado capta o esforço em manter e ampliar o serviço de fornecimento de água tratada. Considerou-se que os efeitos do investimento realizado sobre a redução de endemias tenham uma defasagem de um ano.

Os dados relacionados ao serviço de fornecimento de água tratada foram obtidos junto ao MINISTÉRIO DAS CIDADES (2008), a partir do Sistema Nacional de Informações sobre Saneamento. 
Com o intuito de minorar o problema da baixa discriminação no modelo DEA, foram desconsiderados os municípios que apresentaram valor nulo para alguma das variáveis apresentadas. Diante disto, analisou-se a eficiência de 93 municípios tocantinenses.

A construção de índices de eficiência foi realizada a partir do programa computacional Sistema Integrado de Apoio à Decisão - SIAD, versão 3. A análise de clusters foi realizada a partir do programa computacional Stata, versão 10.0.

\section{Apresentação e Análise dos Resultados}

A Tabela 1 apresenta os índices de eficiência dos dez municípios mais eficientes seguidos pelos dez municípios menos eficientes segundo o modelo DEACCR, bem como o número total de casos das endemias consideradas para que o município seja considerado eficiente. Os municípios foram ordenados com base no índice de eficiência clássico, seguido do índice de eficiência normalizado. O modelo DEA-CCR apresentou cinco municípios na fronteira de eficiência. Verifica-se que o município de Paranã é o mais eficiente e o município de Palmas é o menos eficiente, considerando a medida de eficiência clássica. O município de Palmas, por exemplo, deveria apresentar menos de um caso das endemias consideradas (0,55 contra os 2.633 efetivamente observados) para que fosse considerado eficiente.

Tabela 1 - Os 10 Municípios mais Eficientes e os 10 Municípios menos Eficientes no Modelo DEA-CCR

\begin{tabular}{|c|c|c|c|c|c|c|}
\hline Municípios & $\mathrm{Ef}_{\text {clássica }}$ & Ef $_{\text {invertida }}$ & $\mathrm{Ef}_{\text {composta }}$ & Ef $_{\text {normalizada }}$ & $\begin{array}{c}\text { Número de } \\
\text { Casos de } \\
\text { Endemias - } \\
\text { Real } \\
\end{array}$ & $\begin{array}{c}\text { Número de } \\
\text { Casos de } \\
\text { Endemias - } \\
\text { Alvo } \\
\end{array}$ \\
\hline Paranã & 1,000000 & 0,000209 & 0,999896 & 1,000000 & 1 & 1,00 \\
\hline Palmeirante & 1,000000 & 0,000222 & 0,999889 & o,999993 & 1 & 1,00 \\
\hline Bernardo Sayão & 1,000000 & 0,000258 & 0,999871 & 0,999975 & 1 & 1,00 \\
\hline Maurilândia do Tocantins & 1,000000 & 0,000643 & 0,999678 & 0,999783 & 2 & 2,00 \\
\hline Sandolândia & 1,000000 & 0,000709 & 0,999645 & 0,999750 & 1 & 1,00 \\
\hline Filadélfia & 0,999506 & 0,000834 & 0,999336 & 0,999440 & 3 & 3,00 \\
\hline $\begin{array}{l}\text { Marianópolis do } \\
\text { Tocantins }\end{array}$ & 0,902882 & 0,000275 & 0,951304 & 0,951403 & 1 & 0,90 \\
\hline São Bento do Tocantins & 0,899369 & 0,000619 & 0,949375 & 0,949474 & 2 & 1,80 \\
\hline Lizarda & 0,886807 & 0,000272 & 0,943268 & 0,943366 & 1 & 0,89 \\
\hline Rio da Conceição & 0,785106 & 0,000375 & 0,892366 & 0,892459 & 1 & 0,79 \\
\hline Almas & o,o03779 & 0,067785 & 0,467997 & 0,468046 & 219 & 0,83 \\
\hline Santa Rosa do Tocantins & 0,003225 & 0,159413 & 0,421906 & 0,421950 & 323 & 1,04 \\
\hline Alvorada & 0,002654 & 0,083449 & 0,459602 & 0,459650 & 197 & 0,52 \\
\hline Colinas do Tocantins & 0,002097 & 0,268298 & 0,366899 & 0,366938 & 289 & 0,61 \\
\hline Miracema do Tocantins & 0,001405 & 0,155156 & 0,423124 & 0,423168 & 456 & 0,64 \\
\hline Porto Nacional & 0,000793 & 0,401683 & o,299555 & 0,299586 & 727 & 0,58 \\
\hline Araguaína & 0,000638 & 0,381418 & 0,309610 & 0,309643 & 941 & 0,60 \\
\hline Gurupi & 0,000586 & 0,380950 & 0,309818 & 0,309850 & 924 & 0,54 \\
\hline Paraíso do Tocantins & 0,000460 & 0,467828 & 0,266316 & 0,266344 & 1053 & 0,48 \\
\hline Palmas & 0,000209 & 1,000000 & 0,000104 & 0,000104 & 2633 & 0,55 \\
\hline
\end{tabular}

Fonte: Elaboração própria a partir dos resultados gerados pelo programa computacional SIAD. 
A Tabela 2 apresenta os índices de eficiência dos dez municípios mais eficientes seguidos pelos dez municípios menos eficientes segundo o modelo DEABCC, bem como o número total de casos das endemias consideradas para que o município seja considerado eficiente. Os municípios foram ordenados com base no índice de eficiência clássico, seguido do índice de eficiência normalizado. $\mathrm{O}$ modelo DEA-BCC apresentou dezoito municípios na fronteira de eficiência, mais que o triplo de municípios observados no modelo DEA-CCR ${ }^{15}$. Observa-se que todos os municípios eficientes no modelo DEA-CCR também o são no modelo DEA-BCC, mas não na mesma ordem, seguindo o critério de eficiência padronizada. O município de Palmas não configura entre os dez municípios mais ineficientes ${ }^{16}$

Tabela 4 - Os 10 Municípios mais Eficientes e os 10 Municípios menos Eficientes no Modelo DEA-BCC

\begin{tabular}{|c|c|c|c|c|c|c|}
\hline Municípios & $\mathrm{Ef}_{\text {clássica }}$ & Ef $f_{\text {invertida }}$ & $\mathrm{Ef}_{\text {composta }}$ & $\mathrm{Ef}_{\text {normalizada }}$ & $\begin{array}{c}\text { Número de } \\
\text { Casos de } \\
\text { Endemias - } \\
\text { Real } \\
\end{array}$ & $\begin{array}{c}\text { Número de } \\
\text { Casos de } \\
\text { Endemias - } \\
\text { Alvo } \\
\end{array}$ \\
\hline Arraias & 1,000000 & 0,001516 & 0,999242 & 1,000000 & 1 & 1,00 \\
\hline Paranã & 1,000000 & 0,001574 & 0,999213 & 0,999971 & 1 & 1,00 \\
\hline Bernardo Sayão & 1,000000 & 0,003120 & 0,998440 & 0,999198 & 1 & 1,00 \\
\hline Palmeirante & 1,000000 & 0,003954 & 0,998023 & 0,998780 & 1 & 1,00 \\
\hline Sandolândia & 1,000000 & 0,003987 & 0,998007 & 0,998764 & 1 & 1,00 \\
\hline Filadélfia & 1,000000 & 0,005359 & 0,997321 & 0,998077 & 3 & 3,00 \\
\hline $\begin{array}{l}\text { Maurilândia do } \\
\text { Tocantins }\end{array}$ & 1,000000 & 0,008256 & 0,995872 & 0,996628 & 2 & 2,00 \\
\hline Rio da Conceição & 1,000000 & 0,009464 & 0,995268 & 0,996023 & 1 & 1,00 \\
\hline São Bento do Tocantins & 1,000000 & 0,010031 & 0,994984 & 0,995739 & 2 & 2,00 \\
\hline Piraquê & 1,000000 & 0,011673 & 0,994164 & 0,994918 & 3 & 3,00 \\
\hline Cristalândia & 0,007444 & 0,223443 & 0,392001 & 0,392298 & 90 & 0,67 \\
\hline Gurupi & 0,007192 & 0,491718 & 0,257737 & 0,257933 & 924 & 6,65 \\
\hline Figueirópolis & o,005965 & 0,338686 & 0,333640 & o,333893 & 99 & 0,59 \\
\hline Porto Nacional & 0,005916 & 0,534878 & 0,235519 & 0,235698 & 727 & 4,30 \\
\hline Pium & 0,005661 & 0,363792 & 0,320935 & 0,321178 & 97 & 0,55 \\
\hline Miracema do Tocantins & 0,005651 & 0,349572 & 0,328039 & 0,328288 & 456 & 2,58 \\
\hline Natividade & 0,005568 & 0,293374 & 0,356097 & 0,356367 & 163 & 0,91 \\
\hline Alvorada & 0,004858 & 0,393446 & 0,305706 & 0,305938 & 197 & 0,96 \\
\hline Paraíso do Tocantins & 0,003667 & 0,730333 & 0,136667 & 0,136771 & 1053 & 3,86 \\
\hline Santa Rosa do Tocantins & 0,003484 & 1,000000 & 0,001742 & 0,001743 & 323 & 1,13 \\
\hline
\end{tabular}

Fonte: Elaboração própria a partir dos resultados gerados pelo programa computacional SIAD.

O município de Arraias é o mais eficiente e o município de Santa Rosa do Tocantins é o menos eficiente, considerando a medida de eficiência clássica. Tomando o município de Santa Rosa do Tocantins como exemplo, este deveria apresenta um pouco mais de um caso das endemias consideradas $(1,13$, contra os 323 casos efetivamente observados) para que fosse considerado eficiente.

\footnotetext{
15 Já era de se esperar que o modelo DEA-BCC apresentasse mais DMUs eficientes do que o modelo DEA-CCR. Este resultado pode ser visto a partir do Gráfico 2.1

${ }^{16} \mathrm{Na}$ verdade, o município de Palmas é o $11^{\circ}$ município mais ineficiente no modelo DEA-BCC.
} 
Em ambos os modelos DEA, verifica-se o fato de que os municípios mais ineficientes devem reduzir drasticamente os casos de endemias para que atinjam a fronteira de eficiência.

Conforme afirmado anteriormente, a existência de outlilers pode afetar os índices de eficiência, em especial considerando-se o DEA-CCR. No caso da eficiência do combate às endemias por meio dos serviços de saneamento, é possível que municípios que apresentem melhores serviços de atendimento hospitalar apresentem baixos índices de eficiência. Observe que esses municípios atraem um maior número de pessoas para o tratamento de endemias ${ }^{17}$. Com base nas variáveis utilizadas nas análises dos modelos DEA-CCR e DEA-BCC foi construído o dendrograma apresentado no Gráfico 4 com o intuito de identificar possíveis outliers.

Gráfico 4 - Dendrograma dos Municípios Tocantinenses Analisados segundo as Variáveis Utilizadas na Análise do DEA: 10 Grupos

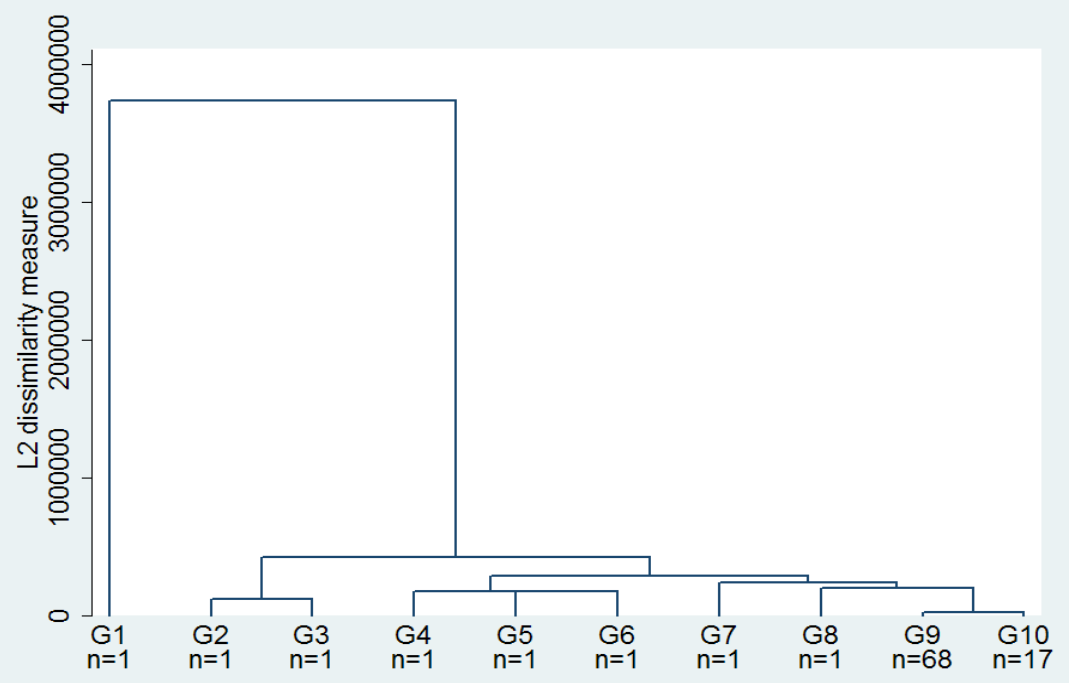

Fonte: Elaboração própria a partir dos resultados gerados pelo programa Stata 10.1.

Os grupos de 1 a 8 compõem, respectivamente, os municípios: Palmas, Colinas do Tocantins, Porto Nacional, Gurupi, Araguaína, Paraíso do Tocantins, Peixe e Guaraí18. A partir dos resultados apresentados no dendrograma, estes municípios podem ser considerados como outliers. É possível observar a partir do Gráfico 4 que, em geral, os municípios considerados outliers estão mais próximos dos grupos 9 e 10 do que uns dos outros ${ }^{19}$. Isso indica que estes municípios não podem ser tratados como pertencentes ao mesmo grupo. Verifica-se que o município de Palmas apresenta uma grande discrepância em relação aos demais municípios analisados. Considerando os índices clássicos de eficiência de ambos os modelos DEA, a retirada

\footnotetext{
${ }_{17}$ Este problema pode persistir mesmo quando se considera as endemias por local de residência, como é feito nesta pesquisa. A persistência do problema ocorre por causa de comportamentos estratégicos por parte dos indivíduos. Os indivíduos têm incentivos para mentir sobre o local onde residem, haja vista que isso pode facilitar seu atendimento médico.

${ }_{18}$ A Tabela A9, em anexo, apresenta a classificação de todos os municípios analisados com base na análise de clusters.

19 As exceções são: os municípios de Colinas do Tocantins e Porto Nacional (G2 e G3) e os municípios de Gurupi, Araguaína e Paraíso do Tocantins (G4, G5 e G6).
} 
dos outliers não implica em mudanças, haja vista que nenhum dos municípios outliers atingiu a fronteira de eficiência.

\section{Considerações Finais}

Diante das evidências do efeito benéfico dos serviços de saneamento sobre a Saúde Pública, torna-se necessário que o planejamento e a execução de políticas de saúde e de intervenções a partir do saneamento devem ser considerados de forma mutua e compassada, de modo a constituir uma estratégia de Saúde Pública preventiva e social. Neste sentido, os resultados obtidos neste estudo possuem grande relevância, haja vista que fornecem um panorama das condições em que os serviços de saneamento estão sendo ofertados nos municípios tocantinenses.

Os índices de eficiência obtidos a partir do método DEA permitiram comparar os municípios quanto a sua eficiência no combate às doenças de veiculação hídrica por meio do saneamento básico. Tal comparação possibilitou não só a identificação dos municípios eficientes e ineficientes, mas também a classificação dos municípios e a identificação dos benchmarks.

Os modelos DEA-BCC apresentaram maiores números de municípios eficientes quando comparado com os modelos DEA-CCR equivalentes. Este resultado se deriva da restrição adicional adotada no PPL do DEA-BCC. Desta forma, a fronteira de eficiência construída pelo método DEA-BCC é mais flexível do que a fronteira construída pelo método DEA-CCR.

A análise de clusters forneceu evidência de que os municípios de: Palmas, Colinas do Tocantins, Porto Nacional, Gurupi, Araguaína, Paraíso do Tocantins, Peixe, Guaraí são outliers em relação aos demais municípios analisados, considerando as características expressas pelas variáveis utilizadas na construção das fronteiras de eficiência. Adicionalmente, também foi possível constatar que estes municípios não podem ser tomados como pertencentes ao mesmo grupo.

Foi observado, ainda, que os municípios considerados como outliers apresentaram os menores índices de eficiência em ambos os modelos DEA. Considera-se a hipótese de que a ineficiência dos municípios outliers se deve ao fato de apresentarem melhores serviços de atendimento hospitalar, atraindo um número maior de indivíduos para o tratamento de endemias, sendo estes municípios pólos de atendimento hospitalar. Dessa forma, a comparação global dos municípios tocantinenses não seria possível ${ }^{20}$.

Adicionalmente, observa-se que o controle metodológico de adotar os casos de endemias por local de residência do paciente pode apresentar limitações, considerando o comportamento estratégico dos indivíduos ${ }^{21}$. $\mathrm{O}$ resultado seria um número superestimado de internações para os municípios com melhores atendimentos hospitalares.

Como nenhum dos municípios outliers atingiu quaisquer fronteira de eficiência, sua retirada não afeta os índices de eficiência clássica. Cabe ressaltar que a ineficiência observada pelo modelo DEA-CCR pode ser resultado do fato do município não operar com rendimentos constantes de escala.

\footnotetext{
${ }^{20}$ Cabe ressaltar que esta hipótese não foi testada na pesquisa, não fazendo parte dos objetivos.

${ }^{21}$ Conforme mencionado anteriormente, os indivíduos que tratam de doenças fora do município de domicílio têm incentivos para mentir sobre o local onde residem, visando um atendimento hospitalar mais ágil.
} 
Em relação ao combate de doenças de veiculação hídrica, observou-se que os municípios considerados mais ineficientes teriam que reduzir de forma drástica os números de casos para que atingissem a fronteira de eficiência.

\section{Referências}

BUSS, Paulo Marchiori. Promoção da Saúde e Qualidade de Vida. Ciência \& Saúde Coletiva, Rio de Janeiro, vol. o5, $\mathrm{n}^{0}$ 01, 2000. p. 163-177.

CARMO, Cinthya M.; TÁVORA JUNIOR, José Lamartine. Avaliação da eficiência técnica das empresas de saneamento brasileiras utilizando a metodologia DEA. Encontro Nacional de Economia (ANPEC) 32, 2004. Anais. João Pessoa: ANPEC, 2004. 1 CD-ROM.

CHARNES, A.; COOPER, W. W.; LEWIN, A. Y.; SEIFORD, Lawrence L. M. Data Envelopment Analysis: theory, methodology and applications. Dordrecht: Kluwer Academic Publishers, 1994.

FUNDAÇÃO NACIONAL DE SAÚDE (FUNASA). Manual de saneamento. 3 . ed. Rev. - Brasília: Fundação Nacional de Saúde, 2006.

GOMES, Adriano Provezano; BAPTISTA, Antônio José Medina dos Santos. Análise Envoltória de Dados: conceitos e modelos básicos. In: SANTOS, Maurinho Luiz dos; VIEIRA, Wilson da Cruz. Métodos Quantitativos em Economia. Viçosa: UFV, 2004.

HELLER, Léo. Relação entre Saúde e Saneamento na Perspectiva do Desenvolvimento. Ciência \& Saúde Coletiva, Rio de Janeiro, vol. 03, nº 02, 1998. p. $73-84$.

KUPFER, David; HASENCLEVER, Lia. Economia Industrial: fundamentos teóricos e práticas no Brasil. Rio de Janeiro: Campus, 2002.

LETA, Fabiana Rodrigues; MELLO, João Carlos Correia Baptista Soares de; GOMES, Eliane Gonçalves; MEZA, Lídia Angulo. Métodos de Melhora de Ordenação em DEA aplicados à Avaliação Estática de Tornos Mecânicos. Investigação Operacional, Lisboa, vol. 25, n⿳0 02, 2005. pp. 229-242.

LINS, Marcos Pereira Estellita; CALÔBA, Guilherme Marques. Programação linear com aplicações em teorias dos jogos e avaliação de desempenho. Rio de Janeiro: Editora Interciência, 2006.

LINS, Marcos Pereira Estellita; MEZA, Lídia Angulo. Analise envoltória de dados e perspectivas de integração no ambiente do apoio à decisão; Rio de Janeiro: COPPE/UFRJ, 2000.

MELLO, João Carlos Correia Baptista Soares de; MEZA, Lídia. Angulo; GOMES, Eliane. Gonçalves; BIONDI NETO, Luiz. Curso de Analise Envoltória de Dados. XXXVII Simpósio Brasileiro de Pesquisa Operacional (SBPO). GramadosRS, 2005. 
MINISTÉRIO DAS CIDADES. Sistema Nacional de Informações sobre Saneamento- SNIS. Disponível em: http://www.snis.gov.br/. Acesso em: 28 de maio de 2008.

MINISTÉRIO DA SAÚDE. Sistema de Informação de Agravos de Notificação - SINAN. Disponível em: http://dtr2004.saude.gov.br/sinanweb/novo/\#. Acesso em: 28 de maio de 2008.

PINDYCK, Robert S.; RUBINFELD, Daniel L. Microeconomia. $5^{\text {a }}$ ed. São Paulo: Prentice Hall, 2002.

MINGOTI, Sueli Aparecida. Análise de Dados através de Métodos de Estatística Multivariada: uma abordagem aplicada. Belo Horizonte: Editora UFMG, 2005.

SANTOS, Nelson Rodrigues dos. Desenvolvimento do SUS, Rumos Estratégicos e Estratégias para Visualização dos Rumos. Ciência \& Saúde Coletiva, Rio de Janeiro, vol. 12, $\mathrm{n}^{\mathrm{O}}$ 02, 2007. p. 429-435.

SANTOS, Jair Lício Ferreira; WESTPHAL, Márcia Faria. Práticas Emergentes de um Novo Paradigma de Saúde: o papel da Universidade. Estudos Avançados, São Paulo, vol. 13, nº 05, jan./apr., 1999. pp. 71-88.

SISTEMA NACIONAL DE INFORMAÇÕES SOBRE SANEAMENTO - SNIS. Diagnóstico dos serviços de água e esgoto - 2000. Disponível em: http:/www.snis.gov.br. Acesso em 17 de abril de 2007a.

SISTEMA NACIONAL DE INFORMAÇÕES SOBRE SANEAMENTO - SNIS. Diagnóstico dos serviços de água e esgoto - 2005. Disponível em: http:/www.snis.gov.br. Acesso em 17 de abril de 2007b.

SOARES, Sérgio R. A.; BERNARDES, Ricardo S.; CORDEIRO NETTO, Oscar de M. Relações entre Saneamento, Saúde Pública e Meio Ambiente: elementos para a formulação de um modelo de planejamento em saneamento. Caderno de Saúde Pública, Rio de Janeiro, vol. 18, nº 06, 2002. pp. 1713-1724.

VARIAN, Hall R. Microeconomic Analysis. 3rd. edition. New York: W.W. Norton and Co, 1992. 506p.

VARIAN, Hal R. Microeconomia: princípios básicos. $6^{\mathrm{a}}$ ed. Rio de Janeiro: Campus, 2003.

VASCONCELLOS, Marco Antônio Sandoval de; OLIVEIRA, Roberto Guena. Microeconomia. ${ }^{\text {a }}$ ed. São Paulo: Atlas, 2006.

UNIVERSIDADE IMES. Principais Doenças Relacionadas com a Água. Disponível em: http://www.imesexplica.com.br/020905agua_doencas.asp. Acesso em: 03 de maio de 2008. 